\title{
Prevalencia y factores de riesgo de sobrepeso y obesidad en escolares de la provincia de Imbabura. Ecuador. 2010.
}

\author{
Overweight and obesity prevalence and risk \\ factors in schoolchildren. Imbabura \\ province. Ecuador. 2010.
}

\begin{abstract}
The objective of this study was to determine the risk factors for overweight and obesity in children of Imbabura province (Ecuador). In a sample with random selection 450 students $6-12$ years of age were chosen. In 22 urban and rural schools we evaluated overweight and obesity with Body Mass Index (BMI) for age, arm skinfold and shoulder and abdomen circumference. We define overweight with BMI for age between 85 to 95 percentile and obesity above percentile 95 and obesity was confirmed by measurement of skinfold above percentile 90. We assessed energetic intake, physical activity and socio demographics aspects by a questionnaire. The prevalence of overweight and obesity was $13.6 \%$ (10\% overweight and $3.6 \%$ obese). This problem was higher in children from private schools. Average daily energy ingest was 2,195 Kcal. Poor physical activity was another associated factor, $25.1 \%$ of assessed children watched over 3 hours of television daily but we didn't find association between television use and overweight and obesity. Logistic regression analysis shows risk factor for overweight and obesity the preference of drink soda (OR 2.7 IC 95\% 1, 3-5.3), sons of mothers with remunerated job (OR 2.5, IC 95\% 1.3-4.8), poor physical activity (OR 1.6 IC 95\% 1.2-2.2) and go to school by some kind of transport ( OR 2.0 IC 95\% 1.4-3.0); the preference for orange juice was a factor of protection ( ORO, 3 IC 95\% 0.15-0.8).
\end{abstract}

Key words: overweight, obesity, BMI/ age, physical activity.

\section{INTRODUCCIÓN}

El Índice de Masa Corporal (IMC) es el indicador recomendado por la Organización Mundial de la Salud (OMS) para evaluar antropométricamente, el estado nutricional de una población menor de 20 años, por su sencillez, bajo costo y adecuada correlación con la grasa corporal total. Sin embargo, para el diagnóstico de obesidad durante la pubertad mediante este indicador, es recomendable, para mejorar su sensibilidad, considerar la raza, el sexo y el grado de desarrollo puberal alcanzado (1).

La diferencia entre sobrepeso y obesidad radica en la cantidad de grasa almacenada, siendo mayor en la obesidad. Esta enfermedad puede presentarse tanto en individuos pobres como en ricos, pero con características diferentes. Estas diferencias pueden deberse a factores adaptativos, alimentarios, socioculturales y de género (2).
Mariana Oleas G.

Universidad San Carlos de Guatemala. INCAP. Guatemala.

Dirigir la correspondencia a: Profesora Mariana Oleas $G$

Nutricionista MSc. en Alimentación y Nutrición

Universidad San Carlos de Guatemala. INCAP. Docente investigadora de la Universidad Técnica del Norte. Avenida 17 de Julio 5-21 Ciudad Universitaria Barrio el Olivo. Guatemala. Teléfono: 0998986183

Este trabajo fue recibido el 24 de Septiembre de 2013 y aceptado para ser publicado el 18 de Enero de 2014.
La prevalencia de sobrepeso y obesidad, en la infancia y adolescencia, está aumentando tanto en los países desarrollados, como en los países en vías de desarrollo. Los problemas nutricionales de los escolares, por lo tanto, además de aquellos ocasionados por sobrepeso o desnutrición, se relacionan con malnutrición asociada a hábitos alimentarios poco saludables (3).

En un estudio realizado en Ecuador con 2829 estudiantes hombres y mujeres, se encontró que el $21 \%$ de los adolescentes presentaron exceso de peso $(13,7 \%$ sobrepeso y $7,5 \%$ obesidad), siendo este parámetro mayor en la costa $(24,7 \%)$ que en la sierra (17,7\%), P=0.001. El exceso de peso fue mayor en los colegios privados (25,3\%), que en los colegios públicos (18,9\%), $\mathrm{P}=0.001$, y más frecuente en mujeres (21,5\%), que en hombres $(20,8 \%)$. Los investigadores confirmaron que los niños y adolescentes consumen pocas frutas y verduras, 
hallazgos similares a este estudio, mientras que el consumo de grasas, sobre todo aceite de palma africana (grasas saturadas) es alto.

En relación al consumo de refrigerios y bebidas, en las escuelas y colegios, los datos obtenidos indican que los alimentos consumidos son altamente energéticos y de precio bajo.

La recomendación de los autores refleja la necesidad de inculcar el hábito de consumo de alimentos hipocalóricos como frutas, verduras y hortalizas con el objeto de disminuir el riesgo de sobrepeso y obesidad (4). Tomado de Yépez y colaboradores (2008), así como Montalvan (informe personal), Alvear (informe personal) y Segarra.

La provincia de Imbabura-Ecuador, no dispone de información sobre la prevalencia de sobrepeso y obesidad en escolares, por lo cual, se determinó la necesidad de identificar la magnitud del sobrepeso y obesidad y los factores de riesgo en escolares, cuyos resultados serán utilizados para planificar programas de prevención con diferentes sectores de la sociedad.

\section{SUJETOS Y MÉTODOS}

Diseño de estudio: es un estudio analítico, aleatorio y transversal.

Población y muestra: De 58.785 escolares matriculados en 459 escuelas durante el período escolar 2008-2009, de las Direcciones de Educación Hispana y Bilingüe, mediante una muestra estratificada y aleatoria se seleccionaron 450 escolares, entre 6 a 12 años de edad, de 22 escuelas. La muestra se estimó por cantón, parroquias urbanas y rurales, tipo de escuela, número de escolares y grado. En el caso de niños entre 6 a 9 años, la información fue proporcionada por sus padres. Para este estudio se obtuvo el consentimiento informado de las autoridades de las escuelas y de los padres de los niños.

Variables: las variables medidas fueron los aspectos sociodemográficos, inactividad física, ingesta energética y el sobrepeso y obesidad.

Variables sociodemográficas: La edad del escolar se calculó en base a los registros que constan en la escuela. El nivel de instrucción, ocupación, estado civil de los padres y condiciones de la vivienda, se obtuvo mediante entrevista a los padres, previo consentimiento informado.

Inactividad física: Esta variable se midió cualitativamente mediante un cuestionario desarrollado por el INCAP5. Dado que el tiempo dedicado a ver televisión, es el principal componente de la inactividad física en escolares, en este estudio se determinó el tiempo dedicado a ver televisión, video juegos, uso del transporte para ir y regresar a la escuela y las tareas que realizan en casa antes de ir y regresar de la misma.

Ingesta energética: Se midió mediante el método de frecuencia de consumo semanal con un cuestionario adaptado para escolares por el Instituto de Nutrición de Centro América y Panamá (INCAP, 2006). Para definir el criterio de patron de consumo se consideraron los alimentos consumidos por más del $25 \%$ de los escolares.

Sobrepeso y obesidad: se evaluó con el IMC de acuerdo a edad y sexo. Los escolares fueron considerados que tenían sobrepeso y obesidad cuando el valor de IMC se situaba por encima de los puntos de corte específicos para su edad y sexo, propuestos por el Centers for Disease Control and Prevention (CDC) de Atlanta. Además, se calcularon puntajes Z para talla/edad con los siguientes puntos de corte: entre menos 2 desviaciones estándar y más 2 desviaciones estándar se consideró normal. Por debajo de menos 2 desviaciones estándar se consideró como retardo en talla o desnutrición crónica. La grasa subcutánea se midió mediante los pliegues tricipital y subescapular (OMS, 1995)6. Los datos obtenidos se compararon con el percentil 90 de los valores de Health Examination Survey y National Health and Nutrition Examination Survey (NHANES) para hombres y mujeres de 9 años en adelante, y para hombres y mujeres de 1 a 74 años de edad, los valores de Frisancho para el pliegue tricipital. Además se midió la circunferencia de la cintura.

Procedimientos para la recolección de información e instrumentos utilizados: Inicialmente se contactó con los Directores Provinciales de Educación Hispana y Bilingüe y se capacitó a los supervisores y directores de las escuelas para obtener su autorización y apoyo. Posteriormente se capacitó a 3 nutricionistas para la recolección de los datos y para las mediciones antropométricas de peso, talla, circunferencia de la cintura, pliegues tricipital, subescapular y porcentaje de grasa de acuerdo a las técnicas recomendadas. El peso se midió en una balanza digital TANITA, con precisión de 100 gramos, la talla se midió en tallímetros de madera con precisión de 0,4 cm, los pliegues con lipocaliper harpender con exactitud de 2 milímetros y la cintura con cintas métricas inextensibles. Todos los equipos fueron estandarizados al inicio de cada medición.

Análisis estadístico: Los datos fueron analizados con el programa Epilnfo. Se calcularon prevalencias de sobrepeso y obesidad utilizando estadísticas descriptivas. Se utilizó el programa Nutrition de Epilnfo 20003.4 con las tablas de referencia de CDC 2000 para calcular percentiles y puntajes $Z$ de los indicadores antropométricos. Se hizo un análisis estadístico univariado, bivariado y de diferencias de medias con todas las variables mediante ANOVA $(p<0,005)$. Con las variables independientes que presentaron valores de $\mathrm{p}$ menor a 0,20 (en el análisis estadístico bivariado), se realizó un análisis multivariado con el paquete informático Stat View.

\section{RESULTADOS}

De los 450 escolares, 50,4\% fueron hombres y $49,6 \%$ fueron mujeres, distribuidos entre las edades de 6 a 12 años o más. En su mayoría, fueron hijos de padres jóvenes con bajo nivel de instrucción, especialmente la madre, cuya ocupación principal fue los quehaceres domésticos y la agricultura, seguida por las actividades comerciales, en el caso del padre. El $63,6 \%$ de familias vivían en casa propia, el 65,8 \%, cuentan con agua potable y el $77,6 \%$ disponen de servicio higiénico. El 70,4\% de los padres daban dinero a sus hijos para la compra de alimentos en la escuela. El 37,1 \% de padres mencionaron que no tenían control sobre lo que comen en la escuela y 35,3 $\%$ tenían la costumbre de premiar a sus hijos con alimentos.

El 50,4\% de los escolares no ayudan a desarrollar actividades en el hogar antes de ir a la escuela y 40,7\% utilizan cualquier tipo de transporte para ir y regresar de la escuela. En relación al tiempo utilizado en ir y volver de la escuela, se encontró que $24 \%$ no caminan y $71 \%$ lo hacen por media hora. El 88,4\% de familias tienen televisión (TV), de éstas, cuatro de cada 10 escolares disponen de TV en su dormitorio, dos de cada 10 niños disponen de TV por cable, el 25\% se dedican a juegos electrónicos, de los cuales 6,9 \% lo hacen todos los días, 18,7\% algunos días de la semana, sin especificar el tiempo que le dedicaron. El 25,1\% mira televisión más de 3 horas diarias los días que van a la escuela, cifra que se duplica (50\%) los días que no van a la escuela. El $15 \%$ de familias siempre acostumbran a comer frente al televisor y el $60 \%$ de los padres no han definido reglas en sus hogares acerca del tiempo de ver televisión.

El patrón de consumo está constituido por 12 alimentos, 
entre los que sobresalen: arroz, azúcar, fideos, papas cocidas y fritas. La fruta de mayor consumo es el banano y la cebolla es la única verdura que utilizan para condimentar las preparaciones. El 50\% de los escolares consumen pollo frito de los cuales 44\% lo hacen cada día. El 70\% toman leche de vaca y con una frecuencia diaria el $21,3 \%$, alimentos que en su mayoría aportan hidratos de carbono, azúcares y grasas, como se observa en la figura 1.

Llama la atención al consumo del café sin y con leche, de los cuales $66,2 \%$ lo hacen sin leche y diariamente.

En relación a las percepciones que los escolares tienen sobre los alimentos, se encontró que la colación que dan en la escuela les gusta mucho $(60,2 \%)$ lo que consistía en galletas y coladas de sabores donadas por el gobierno del Ecuador. El 84,4\% manifestó que le gustaría comer verduras y frutas, $75 \%$ preferían tomar gaseosas en lugar de jugos de naranja o de otras frutas, $72 \%$ preferían el consumo de "chitos" en relación con las frutas. Además, $80,7 \%$ señalaron que les gusta mucho la clase de educación física y realizan deporte el $30 \%$. A pesar que el $66 \%$ utilizan su tiempo mirando la televisión, manifestaron que el juego es un componente importante en su vida, sin especificar el tipo de juego y el tiempo que le dedican.
En la tabla 1, se observa que 7,3\% presentan bajo peso, $10 \%$ sobrepeso y $4,0 \%$ obesidad. Estos resultados sugieren que existen problemas nutricionales por déficit y exceso que inducen al bajo peso, sobrepeso y obesidad, y que a futuro constituirían un problema de salud pública en la provincia de Imbabura.

Entre los hallazgos se encontró que la obesidad y el sobrepeso fueron mayores en las escuelas particulares, 10,3\%, $13,5 \%$, respectivamente y menor en las fiscales, $0,93 \%$ y $8,6 \%$, en su orden $(p<0,0001)$.

Retardo de talla presentó el 22,7\%, incrementándose conforme avanza la edad, valor que fue mayor en escolares de escuelas fiscales, independientemente del sexo.

En la tabla 2 se observa que las medias de la circunferencia de la cintura aumentan con la edad en forma significativa $(p<0,0001)$. El valor más alto se encontró en el grupo de 9,1 a 12 años; la frecuencia de escolares con valores de la circunferencia de cintura sobre el percentil 90 fue $6 \%$. La circunferencia de la cintura mostró alta correlación con el IMC $(r=0,8)$. La media mayor de los pliegues tricipital y subescapular se encontró en escolares menores de 6 años, así como el porcentaje de grasa.

Al medir el estado nutricional con el pliegue subescapular,

\section{FIGURA 1}

Alimentos de consumo frecuente.

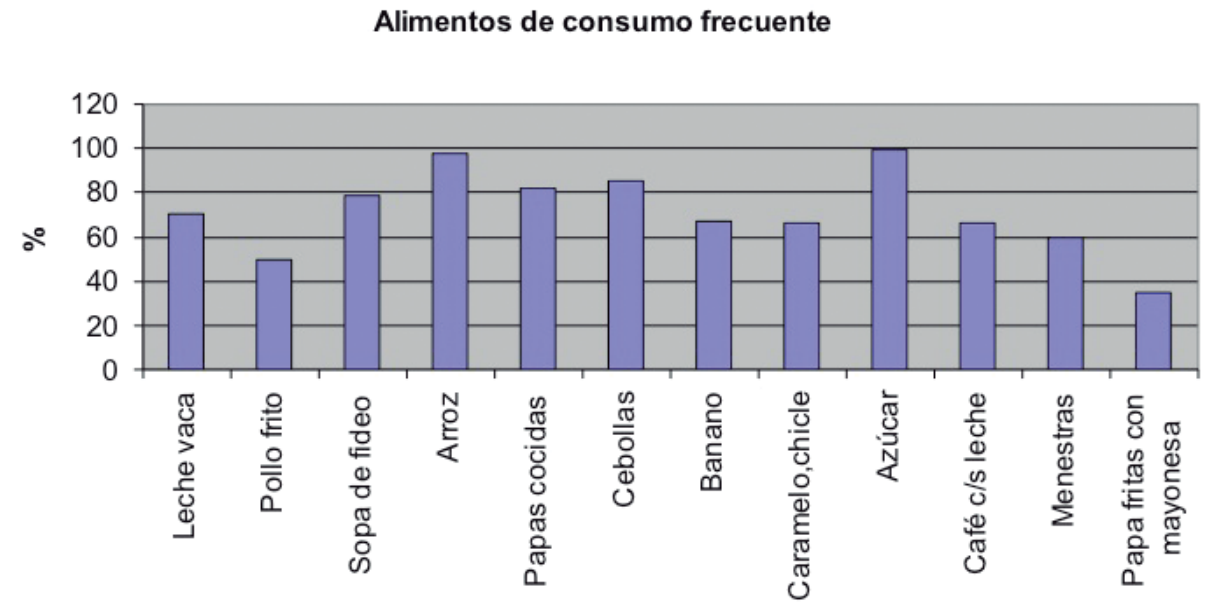

TABLA 1

Estado nutricional de los escolares según el IMC/edad.

\begin{tabular}{|c|c|c|}
\hline Estado nutricional & Número & $\%$ \\
\hline Bajo peso & 33 & 7,3 \\
\hline Normal & 356 & 79,0 \\
\hline Sobrepeso & 45 & 10,0 \\
\hline Obesidad & 16 & 3,6 \\
\hline Total & 450 & 100.0 \\
\hline
\end{tabular}


se encontró que de los 16 escolares clasificados como obesos según IMC, 13 fueron confirmados que son obesos y de los 45 escolares con sobrepeso mediante IMC, 12 fueron catalogados con obesidad. Con el pliegue tricipital, 15 presentaron obesidad y de los 45 escolares con sobrepeso a través del IMC, 20 fueron evaluados con obesidad.

En los niños en edad escolar la circunferencia de la cintura y los pliegues ayudaron a confirmar el diagnóstico que 13,6\% presentaron exceso de peso y obesidad.

La ingesta promedio diaria de energía, se incrementó en los escolares con sobrepeso y obesidad $(2194,9 \pm 1413$ y $2206 \pm 1075$ ). Este incremento fue significativo, y puede ser considerado como el factor de riesgo de sobrepeso y obesidad (tabla 3 ).

En la tabla 4 se observa que la preferencia por el consumo de gaseosas incrementa el riesgo de adquirir sobrepeso y obesidad (OR 2,7); en cambio, la preferencia de jugo de naranja u otra fruta sobre el agua, constituye un factor de protección (OR 0,35). Los hijos de madres con trabajo remunerado tienen mayor riesgo de desarrollar sobrepeso y obesidad (OR 2,6). Finalmente, los niños menos activos y los que se van a la escuela todos los días en algún tipo de transporte comparados con los que llegan caminando a la escuela, también fueron factores de riesgo para adquirir obesidad (OR de 1,7 y 2,1 respectivamente). Llama la atención que el número de horas destinadas a mirar televisión, no fue un factor de riesgo.

\section{DISCUSIÓN}

Los hallazgos de este estudio indican que la prevalencia de sobrepeso y obesidad en escolares de la provincia de Imbabura es similar a la reportada a nivel nacional para niños de la misma edad en áreas urbanas de algunas ciudades ecuatorianas4. Además, estos problemas nutricionales se asocian al patrón de consumo, preferencias alimentarias y la inactividad física de los escolares. El sobrepeso y obesidad fue mayor en las escuelas particulares que en las fiscales. Por otra parte llama la atención en este estudio que la prevalencia de sobrepeso y obesidad, fue similar tanto en hombres como en mujeres, puesto que este problema se ha observado mas en mujeres, tanto en nuestro país como en otros países del mundo.

De acuerdo al modelo de regresión logística, los factores de riesgo de sobrepeso y obesidad en los escolares fueron la preferencia por el consumo de gaseosas, ser hijos de madres con trabajo remunerado, los niños menos activos y los que se van a la escuela todos los días en algún tipo de transporte, mientras que la preferencia por el consumo de jugo de naranja o alguna otra fruta fue un factor protector.

Por otro lado, el estudio reporta, que $22,7 \%$ de los escolares evaluados presentaron retardo en talla, problema que fue mayor en las escuelas fiscales, mientras que a nivel nacional fue del 15,6 \% de escolares que sufrían de desnutrición crónica el año 2005. Una causa frecuente de la obesidad, es el retardo del crecimiento en los primeros años de vida, retardo que no se revierte en la mayoría de los casos en etapas posteriores, por lo cual cuando incrementan su ingesta energética o reducen la actividad física, tienen mayor riesgo de obesidad. Varios estudios realizados en algunos países a nivel de Latinoamérica avalan esta situación $(7,8)$.

Los resultados obtenidos muestran que los escolares son sedentarios debido a que no ayudan a sus padres a desarrollar actividades en el hogar, caminan poco, miran mucha televisión, se dedican a juegos electrónicos y los padres no han definido reglas en sus hogares acerca del tiempo de ver televisión. Si bien, los escolares mostraron altos niveles de tiempo viendo televisión, en este estudio no se encontró una asociación estadísticamente significativa con sobrepeso y obesidad. Según varios estudios, el hábito de ver televisión ha aumentado considerablemente y es una de las causas que se asocia al

TABLA 2

Medias y desviaciones estándar de las medidas antropométricas por grupo de edad de los escolares.

\begin{tabular}{lccccc}
\hline $\begin{array}{l}\text { Grupos de edad } \\
\text { (Años) }\end{array}$ & $\mathrm{N}$ & $\begin{array}{c}\text { Circunferencia cintura } \\
(\mathrm{cm})\end{array}$ & $\begin{array}{c}\mathrm{IMC} \\
\left(\mathrm{kg} / \mathrm{tm}^{2}\right)\end{array}$ & $\begin{array}{c}\text { Pliegue tricipital } \\
(\mathrm{mm})\end{array}$ & $\begin{array}{c}\text { Pliegue subescap } \\
(\mathrm{mm})\end{array}$ \\
& & & & & $\begin{array}{c}\text { Grasa } \\
(\%)\end{array}$ \\
$<6$ años & 10 & $57,22 \pm 14,7$ & $17,23 \pm 2,2$ & $11,96 \pm 3,1$ & $9,02 \pm 5,0$ \\
$6,1-9$ & 189 & $60,24 \pm 7,19$ & $16,46 \pm 1,9$ & $9,93 \pm 3,6$ & $7,25 \pm 3,1$ \\
$9,1-12$ & 223 & $64,11 \pm 6,95$ & $17,36 \pm 2,4$ & $10,99 \pm 4,3$ & $8,44 \pm 4,5$ \\
$>12$ & 28 & $62,55 \pm 5,61$ & $17,33 \pm 1,6$ & $9,73 \pm 2,5$ & $21,70 \pm 7,5$ \\
Total & 450 & & & & $6,81 \pm 1,8$ \\
\hline
\end{tabular}

TABLA 3

Ingesta promedio diaria de energía según el estado nutricional de escolares.

\begin{tabular}{lcccc}
\hline Estado nutricional & Casos & Media & Desviación estándar & Error estándar \\
Bajo peso & 31 & 2043,93 & 809,89 & 150,39 \\
Normal & 358 & 1858,63 & 820,4 & 43,72 \\
Sobrepeso & 45 & 2194,9 & 1413,5 & 213,0 \\
Obesidad & 16 & 2206,5 & 1075,2 & 268,8 \\
\hline $\mathrm{F}=2,607 \mathrm{p}<0,0001$ & & & & \\
\hline
\end{tabular}


problema de la obesidad (9).

Respecto a las condiciones sociodemográficas, las familias de los escolares corresponden en mayor proporción a familias de nivel medio que se caracterizan por disponer de casa propia, servicios básicos, respaldados por recursos económicos de supervivencia, por lo tanto los problemas nutricionales son más complejos, pues la obesidad y el sobrepeso no afectan exclusivamente a los sectores sociales con mayor poder adquisitivo. En una misma familia de bajos ingresos pueden coexistir personas que sufren desnutrición con otras que tienen obesidad y bajo consumo de micronutrientes esenciales (10).

Diversos estudios afirman el papel que han jugado determinantes como la modernización agrícola, la innovación tecnológica en la producción de alimentos, la crisis económica, la globalización y la apertura comercial. Respecto a este último elemento, se señala que la existencia de economías abiertas a la importación, entre otros productos, de alimentos industrializados hipercalóricos con importantes contenidos de grasas y azúcares y bajos en carbohidratos no refinados, ha producido cambios en los patrones alimentarios propiciando el incremento del sobrepeso y la obesidad en amplios segmentos poblacionales (10). Recientes estudios epidemiológicos indican que el consumo frecuente de frutas se asocia con un bajo riesgo de sufrir enfermedades crónicas, por lo tanto se debe incrementar el consumo de estas (11).

De acuerdo a un estudio reportado en México en 407 niños, la obesidad tiene origen multifactorial y se han mencionado factores de riesgo que influyen: genéticos, hormonas como la leptina, grelina, adiponectina que influyen en el apetito, saciedad y distribución de la grasa, además de otros como el estatus económico y el ambiente, igualmente reporta prevalencias de obesidad a nivel mundial en niños y niñas, de $5 \%$ en 1963, 17\% en 2004 y en el último año, hasta de 30\% (12). Este estudio reporta una de las tasas más altas (49.6\%) en obesidad y sobrepeso.

En Cuba, al comparar un estudio provincial sobre crecimiento, desarrollo y estado nutricional de niños y adolescentes entre 0 a 19 años efectuado en Ciudad de La Habana en 1998, con otro similar realizado en 1993, se pudo conocer que durante esos 5 años se incrementó el porcentaje de individuos con exceso de peso para la talla de 9,3 a 13,1\% (1,4 veces) y aquellos con niveles elevados de adiposidad de 12,7 a 21,9\% (1,7 veces), lo que evidencia la necesidad de establecer recomendaciones concretas que permitan la detección temprana de estos pacientes (13).

Este estudio tiene limitaciones que se deberían considerar al interpretar los resultados, el método de frecuencia de consumo de alimentos no incluyó cantidades de alimentos, por lo que las raciones promedio de consumo fueron estimadas. No fue posible medir actividad física con pasómetros.

El sobrepeso y obesidad en los escolares puede ir en aumento en la Provincia de Imbabura, lo que obedece a los cambios en los estilos de vida, sedentarismo motivado por el uso de transporte escolar, que hace que incremente el consumo de alimentos que aportan energía; influyen también los medios de comunicación, la ausencia de los padres en el hogar por cuestiones laborales y la delegación en la responsabilidad de la alimentación a los hermanos mayores o personas encargadas del cuidado del hogar, lo que ha conducido al incremento del consumo de alimentos poco saludables.

\section{RESUMEN}

El presente estudio tuvo como objetivo medir la prevalencia y los factores de riesgo que determinan la aparición de sobrepeso y obesidad en escolares de la Provincia de Imbabura (Ecuador). En una muestra estratificada con selección aleatoria de 450 escolares, de 6 a 12 años de edad, distribuidos en 22 escuelas, se evaluó el sobrepeso y obesidad con el Indice de Masa Corporal (IMC) según edad y se complementó con la medición de los pliegues tricipital, subescapular y la circunferencia de la cintura. Se consideró sobrepeso al IMC/edad entre 85 a 95 percentiles, obesidad mayor al 95 percentil y se confirmó la obesidad con pliegues mayores al percentil 90. A través de un cuestionario se evaluó la ingesta de energía (frecuencia de consumo), actividad física y aspectos sociodemográficos. El sobrepeso y obesidad alcanzó a 13,6\%, del cual $10 \%$, correspondió a sobrepeso y 3,6\% a obesidad. Este problema fue mayor en los niños y niñas de las escuelas particulares. El retardo de talla alcanzó 22, 7 \%. La ingesta promedio diaria de energía fue de $2.195 \mathrm{kcal}$. La falta de actividad física fue otro factor asociado al problema, 25,1\% de escolares miran televisión (TV) más de 3 horas diarias los días que van a la escuela, valor que se duplica los días que no van a la escuela; sin embargo, no se encontró una asociación entre el tiempo que miran televisión con el sobrepeso y la obesidad. Según el modelo de regresión logística, demostraron mayor riesgo de sobrepeso y obesidad los escolares que prefieren el consumo de gaseosas (OR 2,7, IC 95\% 1,3-5,3), los hijos de madres con trabajo remunerado (OR2,5, IC 95\% 1,3-4,8); los escolares mucho menos activos (OR 1,6, IC 95\% 1,2-2,2) y los que se van a la escuela todos los días en algún tipo de transporte (OR 2,0, IC 95\% 1,4- 3,0), mientras que la preferencia por el jugo de naranja fue un factor de protección (OR 0,3, IC 95\% 0,15-0,8).

Palabras clave: Sobrepeso, obesidad, IMC/edad, escolar, factores de riesgo, actividad física.

TABLA 4

Factores de riesgo para el sobrepeso y obesidad.

Factores de riesgo

Preferencias entre gaseosas y jugos

Entre jugo de naranja y agua

Niños de madres con trabajo

Niños comparados con otros niños (activos y no activos)

Niños que usualmente van a la escuela en transporte
OR

2,7

0,35

2,6

1,7

2,1
Intervalo de confianza (95\%)

$1,3-5,3$

0,15-0,8

$1,3-4,8$

$1,2-2,2$

$1,4-3,0$
0,0055

0,0121

0,0038

0,0013

Valor de P

$<0,0001$ 
Agradecimientos: Un agradecimiento especial al Ex Consejo Nacional de Educación Superior (CONESUP), por el financiamiento otorgado, así como a la Universidad Técnica del Norte (UTN), por las facilidades brindadas durante la ejecución de la presente investigación. A la especialista Adriana Delgado por los aportes dados en la redacción de este documento. Coordinadora investigación Facultad Ciencias de la Salud. Universidad Técnica del Norte.

\section{BIBLIOGRAFÍA}

1. Rodríguez $O$ L, Pizarro T. Situación nutricional del escolary adolescente en Chile. Rev Chil Pediatr. 2006; 77 (1): 70-80.

2. Organización Panamericana de la Salud. Informe de la reunión técnica sobre obesidad en la pobreza: situación de América latina. Washington. D.C. OPS. 1996.

3. Aguirre M L, Astudillo C, Le Roy O C. Desafíos emergentes en la nutrición del adolescente. Rev Chil Pediatr. 2010;81 (6): 488-97.

4. Hidalgo Ottolenghi F. Paredes Suarez M. La obesidad como pandemia del siglo XXI: Una perspectiva epidemiológica desde Iberoamerica. Real Academia Nacional de Medicina con colaboración de Fundacion MAPFRE. Madrid España. 2008. Pg 200-201.

5. Instituto de Nutrición de Centroamérica y Panamá (INCAP). Manual de instrumentos de evaluación dietética. Traducción de dietary Assessment Resource. Guatemala. INCAP. 2006.
6. Organización Mundial de la Salud (OMS). Uso e interpretación de la antropometría. Ginebra: OMS-OPS. 1995.

7. Atalah E, Urtega C, Rebolledo A, Delfin S, Ramos R. Prevalencia de la obesidad en escolares de la región de Aysen. Rev Chil Pediatr. 1999; 70(6), p. 208-14.

8. Organización Mundial de la Salud (OMS). Prevención en la niñez y la juventud de las enfermedades cardiovasculares en el adulto. Ginebra 1990.

9. Vader AM, Walters ST, Harris TR, Hoclscher DM. Television viewing and snacking behaviors of fourth and eigth grade school childrens in Texas. Preventing chonics disease. CDC Atlanta. Dis. 2009; 6 (3): A 89.

10. Cardaci D. Obesidad infantil en América Latina: Un desafío para la promoción de la Salud. Global Health Promotion. 2013; 20 (3) p 80-2.

11. Contreras Calderón J. Capacidad antioxidante en frutas Colombianas. Rev Vitae 19. 2012; Supl 29.

12. Hernández-Herrera RJ, Salas-Rubio MG, Saaib-Castillo $J I$, Tamez-Rodríguez VA, Dávila Canales R, Ramírez Vela $H$. Prevalencia de sobrepeso y obesidad en 407 niños en Noreste de México. Med Universitaria. 2011;13 (51). 79-83.

13. Suarez Castillo N, Guerrero Ramírez A, Rodríguez Oropesa K, Flores Martínez C, Tadeo Oropesa I. Prevalencia de la Obesidad en un círculo infantil. Rev Cubana Pediatr. 2010; 82 (2). 